\title{
Searching for the perfect lymphadenectomy
}

\author{
Federico Venuta, Daniele Diso, Erino A. Rendina, Marco Anile \\ Department of Thoracic Surgery, University of Rome Sapienza, Rome, Italy \\ Correspondence to: Federico Venuta, MD. University of Rome Sapienza, Policlinico Umberto I, Viale del Policlinico 155, 00161 Rome, Italy. \\ Email: federico.venuta@uniroma1.it. \\ Provenance: This is a Guest Editorial commissioned by Section Editor Maria Rodriguez (Department of Thoracic Surgery, Brigham and Women's \\ Hospital, Boston, MA, USA). \\ Comment on: Samayoa AX, Pezzi TA, Pezzi CM, et al. Rationale for a Minimum Number of Lymph Nodes Removed with Non-Small Cell Lung \\ Cancer Resection: Correlating the Number of Nodes Removed with Survival in 98,970 Patients. Ann Surg Oncol 2016;23:1005-11.
}

Submitted Apr 12, 2017. Accepted for publication Apr 18, 2017.

doi: $10.21037 / \mathrm{atm} .2017 .05 .03$

View this article at: http://dx.doi.org/10.21037/atm.2017.05.03

It is nowadays well known that accurate pathological staging is crucial for lung cancer patients. However, the role of lymphadenectomy seems to be still an open problem: does it exist a real benefit from it in terms of disease free survival (DFS) and overall survival (OS)? Should we perform a complete dissection of the mediastinum and hilum or sampling could be enough? However, there are also other open questions and one in particular is eliciting interest among thoracic surgeons and oncologists; it regards the topic addressed in a paper published in the Annals of Surgical Oncology last August by Samayoa and colleagues (1).

The aim of that study was to establish whether there is a correlation between the numbers of lymph nodes (LN) removed at the time of operation and OS; they studied a large population of non-small cell lung cancer (NSCLC) patients collected in the National Cancer Data Base during a 10-year study period [2004-2014]. The design of the study was extremely simple, with a comparison of OS between five groups of patients with pathologically negative $\mathrm{LN}$ identified by the number of $\mathrm{LN}$ removed: Group 1, 1 to $4 \mathrm{LN}$; Group 2, 5 to $8 \mathrm{LN}$; Group 3, 9 to $12 \mathrm{LN}$; Group 4, 13 to $16 \mathrm{LN}$ and Group 5, 17 or more. T4 patients were excluded; the surgical procedure ranged from sublobar resections to pneumonectomy. As the authors reported in their "Table 1", the above-mentioned inclusion criteria progressively reduced the number of patients included in the study from more than $1,000,000$ to 98,970 ; thus, less than $10 \%$ of the patients included in the database were eligible for the study, although the number is still large enough to allow statistical analysis.

The study showed an advantage in terms of OS for patients with a higher number of $\mathrm{LN}$ removed, with a cutoff at $10 \mathrm{LN}$; particularly, it was observed that patients with 9 or less LN removed had a $12 \%$ increased risk of death. The conclusions confirmed "a significant correlation between the number of $L N$ pathologically examined and overall survival in NSCLC patients".

These results have already been reported in previous studies with data drawn from the Surveillance Epidemiology and End Results (SEER) database (2-4) and the California Cancer Registry (5), although the number of patients was smaller. In addition to the importance of the number of $\mathrm{LN}$ removed, these studies pointed out the large discrepancy between surgeons in the strategy to assess LN; they concluded that inadequate $\mathrm{LN}$ evaluation is "a major quality gap, for which corrective intervention is warranted" (4). It is impossible to disagree: lymphadenectomy should be certainly standardized. However, some details, also in the paper of Samayoa (1), remain controversial and may limit statistical significance. Before speculating on the potential impact of the number of $\mathrm{LN}$ removed on outcome, we should consider also other variables that are equally important: (I) the site of dissection (hilum and/or mediastinum); (II) the type of lymphadenectomy (complete hilar and mediastinal nodal dissection vs. sampling); and (III) the quality of the material removed (en bloc LN $v$. pieces of LN). In this and in most of the previous studies, a pathologically N0 population of patients was included, but no overall information about the site of lymphadenectomy 
was reported. This approach may favor misunderstanding: what variable really impacts on survival? Most of the studies confirm that the minimum number of $\mathrm{LN}$ to be resected is 10; however, there is a big difference in terms of impact on prognosis if we consider 10 negative hilar LN or 5 negative hilar plus 5 negative mediastinal $\mathrm{LN}$ or 10 negative mediastinal LN.

The second point has been extensively considered in the literature; a recent paper by Darling (6) reports the results of the American College of Surgery Oncology Group Z0030 Trial and seems to definitively clarify that no differences can be appreciated between systematic sampling and extensive mediastinal nodal dissection in terms of DFS and OS. However, 2 years later, a review by the same author provided a different interpretation of that trial (7). In fact, since the eligibility criteria excluded clinical T3 and $\mathrm{T} 4$ tumors and patients with preoperatively positive hilar or mediastinal LN, it could be not correct to consider equivalent the two procedures in patients with advanced disease. $\mathrm{Wu}(8)$ found a statistically significant difference in favor of nodal dissection in a cohort of patients that preoperatively did not receive invasive staging, supporting the impact of this procedure on survival. Furthermore, because both studies showed that there was no increased risk of complications due to mediastinal $\mathrm{LN}$ dissection, this procedure should be preferred.

Regarding the third open question, it seems intuitive that the en bloc LN removal guarantees a better chance to discover micrometastases. Samayoa misses all this information in his paper; it could be considered a strong bias risking invalidating results.

Nodal involvement certainly influences prognosis in NSCLC patients, but it is not the only variable involved: the type of resection (anatomical versus non-anatomical), plays also a crucial role. Furthermore, the type of surgical procedure is often associated with the patient's clinical status: sublobar resections may be reserved to patients with poor functional status and these two factors together bear negatively on outcome, independently from the nodal status. This concept is completely missed in this study, where more than 13,000 patients $(13.3 \%)$ received sublobar resections $[12,608]$ or resections not otherwise specified [596]. Also, the extension of the resection seems related to the number of LN removed; in fact, in the text it is clearly reported that "...patients who underwent less than a lobectomy had a mean of $5.6 L N$ removed, compared with a mean of 9.2 for those who underwent lobectomy or bi-lobectomy, and 12.8 with pneumonectomy $(P<0.001)$ ". It has been demonstrated that the type of parenchymal resection shows an impact on survival: lobectomy should be preferred and segmentectomy may be considered only at early stages, particularly in patients at increased risk; wedge resections are at increased risk of local recurrence. However, in this paper no information clarifies what does it mean "less than lobectomy". In other words, in "less than lobectomy" patients what is the most important factor correlated with OS? The suboptimal resection or the reduced number of LN removed? ... or both? Furthermore, in this study more than 3,000 patients (3.3\% of population) had residual disease (R1); does this group belong to the "less than lobectomy" group? This was another confusing factor. Large sample numbers are not always useful to obtain a high statistical power!

Notwithstanding the role of lymphadenectomy in the management of lung cancer is always emphasized, when large series or database are analyzed it is clear that in our daily surgical activities we act differently from the way we should... or what we write. This huge variability leads to a number of criticisms in the interpretation of data. From the paper of Little (9) published in 2005 few things seem to be changed. Probably the percentage of patients that receive pre- or intraoperative $\mathrm{LN}$ assessment is increased, but international guidelines are not always strictly followed (10). This critical point contributes to the inhomogeneity of data within the database, invalidating the analysis. Much more need to be done to reduce this variability and finally be able to answer the "primordial" question: what is the real role of lymphadenectomy: to cure or to stage?

In conclusion, although with several limitations, the report from Samayoa (1) is stimulating but it requires other studies to be confirmed. An interesting suggestion for our daily surgical activities comes from Ramón Rami-Porta (11) in his 2013 editorial: "Leave no lymph node behind!". In fact, a more accurate routine lymphadenectomy will certainly provide more information with a homogeneous population of patients, and consequent reliable data for future studies.

\section{Acknowledgements}

None.

\section{Footnote}

Conflicts of Interest: The authors have no conflicts of interest to declare. 


\section{References}

1. Samayoa AX, Pezzi TA, Pezzi CM, et al. Rationale for a minimum number of lymph nodes removed with nonsmall cell lung cancer resection: correlating the number of nodes removed with survival in 98.970 patients. Ann Surg Oncol 2016;23:1005-11.

2. Ludwig MS, Goodman M, Miller DL, et al. Postoperative survival and the number of lymph nodes sampled during resection of node-negative non-small cell lung cancer. Chest 2005;128:1545-50.

3. Varlotto JM, Recht A, Nikolov M, et al. Extent of lymphadenectomy and outcome for patients with stage I non-small cell lung cancer. Cancer 2009;115:851-8.

4. Osarogiagbon RU, Ogbata O, Yu X. Number of lymph nodes associated with maximal reduction of long-term mortality risk in pathologic node-negative non-small cell lung cancer. Ann Thorac Surg 2014;97:385-93.

5. Ou SH, Zell JA. Prognostic significance of the number of lymph nodes removed at lobectomy in stage IA non-small cell lung cancer. J Thorac Oncol 2008;3:880-6.

Cite this article as: Venuta F, Diso D, Rendina EA, Anile M. Searching for the perfect lymphadenectomy. Ann Transl Med 2017;5(15):311. doi: 10.21037/atm.2017.05.03
6. Darling GE, Allen MS, Decker PA, et al. Randomized trial of mediastinal lymph node sampling versus complete lymphadenectomy during pulmonary resection in the patient with N0 or N1 (less than hilar) non-small cell carcinoma: results of the American College of Surgery Oncology Group Z0030 Trial. J Thorac Cardiovasc Surg 2011;141:662-70.

7. Osarogiagbon RU, Darling GE. Towards optimal pathologic staging of resectable non-small cell lung cancer. Transl Lung Cancer Res 2013;2:364-71.

8. Wu Y, Huang ZF, Wang SY, et al. A randomized trial of systematic nodal dissection in resectable non-small cell lung cancer. Lung Cancer 2002;36:1-6.

9. Little AG, Rusch VW, Bonner JA, et al. Patterns of surgical care of lung cancer patients. Ann Thorac Surg 2005;80:2051-6.

10. Allen MS. Mediastinal lymph node dissection for non-small cell lung cancer. J Thorac Cardiovasc Surg 2005;130:241-2.

11. Rami-Porta R. Leave no lymph nodes behind! Eur J Cardiothorac Surg 2013;44:e64-5. 